\title{
NET4ECG. An ECG Digital Network
}

\author{
S Lightwood, F Greco, V Lizzio, M Brignole, G Gigli \\ ASL4 Chiavarese, Regione Liguria, Italy
}

\begin{abstract}
The choice of ASL 4 was to develop a global network for ECG digital treatment on SCP-ECG standard, integrating the solution with the Hospital Information System and distributing a real-time service from the Cardiology Department to the whole organization. The system is finalized to reduce the elapsed time between the execution of ECG and validation by a cardiology physicians and to construct a patient based historical database of ECG to permit a real-time comparison of former examinations during the valuation of a new one.
\end{abstract}

\section{Introduction}

ECG is one of the most popular diagnostic procedures used in hospitals and outpatients point of care. Despite its instrumental genesis, it didn't have the same development in digital treatment and in definition of standard data formats as medical imaging. OPENECG, a two years Project funded by European Union, focused its attention on SCP-ECG data formats and there are now the first devices and viewings producers complying with this standard.

The definition of an open and recognized standard from the market made possible to ASL4 to project a system finalized to the digital management of ECG. It has been developed respecting the criteria of adhesion to open standards and in the optical of re-use that for ASL4 is a fundamental choice for Information Systems and their integration with biomedical technologies

The whole integration with the Health Information System of ASL4 Chiavarese (OASIS4 - a solution completely developed by ASL4 during the last 10 years) plans the recording of the examination, the immediate availability to the requiring unit of a diagnosis ("first opinion") produced by an ECG analysis software, the optional validation of a cardiology physician with the return of the whole data to the requiring unit.

The capillarity of the project is well represented by the following numbers :

in ASL4 Chiavarese OASIS4 is active on approximately 600 workstations and used by more or less 1400 of the 1800 workers. NET4ECG will provide the integration of all the ECG devices used by our organization (approximately 70).

0276-6547/05 \$20.00 @ 2005 IEEE

\section{Objectives and technical requirements}

The network connects 4 hospitals and the service will be available also for family physicians and specialists operating outside the hospitals. The annual estimated number of ECG validated by cardiologists is about 35,000 .

The main objectives of the project may be summarized by the following list:

1. Reduction of the elapsed time between the execution of ECG and the return of examination confirmed by a cardiology physician

2. Creation of an ECG's historical database to allow to physicians a real-time comparison with former examinations of every single patient.

3. Storage of the digital data in the EHR (Electronic Health Record) of the patient

4. Reduction of number of evaluation request from operative units to cardiology specialists.

5. Integration of every phase of the process with ASL4 Health Information Systems and contextual elimination of any other communication support (phone, fax or paper ..)

6. Definition of a Cardiologic Virtual Department distributed over all ASLA structures to provide telemedicine services.

The will to develop a digital management system for ECG diagnostics has immediately raised the question of the choice of data format. We wanted it free from proprietary solution and open to maximum portability.

The hints of the European Project "Standard Communication Protocol for Computerized Electrocardiography SCP-ECG (1990-1991)" have begun to be followed by some ECG devices main producers .

The gradual achievement of interoperability between SCP formats used by different producers, and the attention towards such a standard of OPEN-ECG workgroup has confirmed ASL4 Chiavarese in this choice.

Another very important fact, that furthermore determined a significant selection between devices for NET4ECG project, was the need to transfer work-lists to a selected device with all the information needed to link the examination with the requiring clinical event. It's clear that immediate and certain linking of ECG data to 
concerning event is a basic element of a project that plans the whole integration with daily clinical activities (of emergency, hospital, or outpatients areas ...). As first result we found that the availability and the adoption of a standard for such a functionality is yet a difficulty for the whole interoperability of ECG systems and their integration with different health information systems.

Besides the mentioned objective, we have focused the following project requirements:

- Integration of all ASL4 structures (hospital, emergency and outpatient care).

- Maximum use of the technological chances offered by ECG automated analysis software.

- Low impact of the project for the end-user by "transparent" introduction of informatics subsystems needed by NET4ECG

- High security standards to trace step by step the flows of operations and data.

- Modular and scalable subsystems to easily act on every single component for maximum portability and availability.

\section{Methods}

The general architecture of NET4ECG is shown in figure 1 where is evident the geographic distribution of ASL4 and the number of used networks.

The ECG digital management system rules the communications with devices and makes available data and ECG recordings to ASL4 health information system (OASIS4). All ECG devices have in input the concerning work-list and return in output, at the end of examination, the 12-lead ECG recordings in SCP format.

OASIS4 transfers the work-lists to an SCP server (which is deputed to communicate with ECG devices), receives back and makes available to the whole organization the ECG recordings and the report generated by automated ECG analysis software and finally, if required, submits and receives back the report confirmed by a cardiology physician.

The software used by cardiology physicians to analyse ECG recordings (SCP viewer) is installed on selected workstations of both cardiologic departments and structures for outpatients care. Every cardiology specialist, based on his organization profile, is allowed to see his concerning requests for ECG confirmation.

As previously mentioned an essential aspect of the project is real-time work-list supply of ECG devices so that the personal making ECG is requested just to do a selection from a preloaded list. The information supplied to ECG devices is comprehensive of elements (like sex, age, ...) useful for a correct interpretation of ECG results and has attributes to link the returned examinations with the concerning request/event managed by ASL4 health information system.

It's meaningful to notice that the work-lists are refreshed every 120 seconds with rules that may be grouped in the following 2 categories :

Automatic refresh :

The work-lists of devices operating for a selected unit or function are automatically refreshed for areas like hospital admissions (by registration in work-list of every inpatient for the concerning unit), emergency (by registration of all patients from triage to discharge on every ECG assigned to emergency area), outpatients booking (by registration of daily data present on each calendar of the interested structure and assigned ECG device).

\section{On-demand :}

Every work-list on each ECG device may be also refreshed by single request of operators using normal functionalities available beneath OASIS4 modules.

One of the aims of the project was to reduce the number of requests towards cardiology specialist. To this purpose the project provides a centralized interpretation program to supply an immediate 1 st level response for all the 12-Lead ECG returned from the devices. The modular structure of NET4ECG allowed a first activation using Telemed and now we are testing an experimental centralized implementation derived from HES. This experimental implementation has, by now, showed worst results than Telemed and the integration effort that determined the choice of submitting to ECG analysis software SCP files generated on the single device has shown that the signal treatment optimised for a determined SW (Telemed) is not immediately suitable for another solution.

One of the most important requirement of the project was to develop a modular system where every component could be easily centrally managed, maintained, substituted or integrated with solutions of other vendors. To this purpose NET4ECG has considered ECG analysis software like any other component of the project determining to install it on a centralized server and not on each ECG device. The benefits of such a choice are obviously easier management of device park (about 70 devices geographically distributed), easier updates and tunings of the software for better fit cardiologists' expectations and, if required, a simple switch between different products or the parallel use of different solutions.

Test results, by now, showed a good sensitivity of both solutions opposed to a certain dissatisfaction of cardiology physicians concerning the propriety of detailed diagnosis in case of pathological ECGs.

The test is based on a selection of 50 ECG recordings between the ones generated in the emergency department where NET4ECG is already active. Despite a good 
sensitivity, it has been noticed a significant number of indications about pathological ECG that instead have been considered normal by cardiology physicians attending the test. And moreover it was identified a numerous set of examinations really pathological but with wrong diagnosis.

Besides the assignation of each ECG to two main categories (normal and pathological), it has been defined a measurement scale to describe the difference between what was considered correct by the three physicians and the results of ECG analysis software :

A. Correct reports substantially confirmable by a physician without updates

B Correct reports confirmable with limited lexical updates

C Partially correct reports which needs some substantial update.

D Reports substantially mistaken.

We also considered, in order to eliminate the interobserver variability, to compare the reports of analysis software with the evaluations of a commission composed by the same three physicians, with the following results

Test results are summarized by the following tables.

\begin{tabular}{|l|c|c|c|c|}
\hline & \# A & \# B & \# C & \#D \\
\hline Single physician & $46.7 \%$ & $27.3 \%$ & $15.3 \%$ & $10.7 \%$ \\
\hline Commission & $44 \%$ & $24 \%$ & $14 \%$ & $18 \%$ \\
\hline
\end{tabular}

Table 1

If we consider cardiologist judgements as the "gold standard" and the two main categories (normal and pathological) for specificity and sensitivity assessment, we have the following results :

\begin{tabular}{|l|c|c|}
\hline & Specificity & Sensitivity \\
\hline Single physician (avg.) & $61,9 \%$ & $78,9 \%$ \\
\hline Commission & $66,7 \%$ & $95 \%$ \\
\hline
\end{tabular}

Table 2

The results notice the need of tuning and improvement of ECG analysis software to meet physicians expectations and by now it has oriented towards Telemed, keeping on tuning the new solution according to the wide configuration criteria available.

\section{Results}

NET4ECG, after the initial activation of 5 devices on 3 hospital units and 1 emergency unit during 6 months, shows 7241 ECGs for 4309 different events (avg. 1.68 ECGs for event). Time intervals elapsing from the execution of ECG and the availability of recordings and 1 st level response on all the workstations of HIS (about 600) resulted 7.1 minutes (50th percentile) (this value decreases to only 2 minutes if we consider just the emergency area). Time from the request of cardiologist diagnosis and the confirmed response to the requiring unit is 11.9 minutes (50th percentile). The confirmation by cardiologist was required 1114 times on 2067 events coming from non cardiologic units $(54 \%)$. As mentioned above, we will keep on testing and tuning ECG analysis software (with wider sets of ECGs) to better satisfaction of one important requirement of the project.

\section{Discussion and conclusions}

The project on the whole constitutes a fundamental element not only towards the optimisation of ECG management process but also to the creation of an historical ECGs database which will allow the physicians to be in better condition to evaluate the evolution of conditions of their patients.

NET4ECG allows maximum scalability and will be exported towards non-hospital point of care and for structures not directly managed by ASL4 like family physicians, home-care, ambulances, no-profit associations....

As shown before the results confirm that the developed solution has been able to meet the main requirements that our organization has focused, results achieved despite some difficulties that we want to remember as last points of our document.

We had to suffer some delays for the wished extension of the project towards other units. This was due to the need to assure a satisfactory stability of the communication and log system as strong requested by the deep integration with working activities and information system.

The introduction of ECGs automated analysis software was more difficult than expected. The use of such a software would have important chances to orient outpatients and routine ECGs evaluations and to decrease the number of requests of 2 nd opinions towards cardiology specialists. Actually these potential benefits have been only partially achieved and also the best results showed in our test resulted in good specificity but a clear disposition to signal non-existent or unimportant pathologies. This aspect becomes more significant in critical areas like emergency departments where the quality of ECG recording is not always perfect and the presence of disturbed signal are frequent. By now this constitutes a serious limitation to the development of the necessary trust from operators (a trust that was the real objective of our test) and, as a consequence, to the impact that an ECG automated analysis software could have.

\section{References}

[1] CEN Health Informatics,TC251: Standard communications protocol for computer assisted electrocardiography - CEN TC 251/N02-015. Available from: URL: www.centc251.org 
[2] Carpeggiani C, Conforti F, Macerata A. ECG Devices Interoperability in Hospital Environment: a Case Report. $2^{\text {nd }}$ OpenECG Workshop 2004, Berlin, Germany.

[3] Zywietz C, Kraemer M, Fischer R, Widiger B. Integrating the ECG Enterprise - HES-EKG with the Built-in Vital Signs Information Nomenclature. In Murray A(ed), Computers in Cardiology 2004

[4] Willems JL, et al. The diagnostic performance of computer programs for the interpretation of electrocardiograms. The New England Journal of Medicine. December 1991. Vol. 325 1767-73

Address for correspondence

Simone Lightwood, Franco Greco, Vincenzo Lizzio

Dipartimento Informativo e Tecnologico

ASL4 CHIAVARESE

Via G.B. Ghio 9, 16043, Chiavari (ITALY)

slightwood@as14.liguria.it fgreco@as14.liguria.it

vlizzo@as14.liguria.it 